\title{
From Nuclear Medicine to Nuclear Medicine Theranostics
}

\author{
Dong Soo Lee ${ }^{1}$
}

Published online: 20 May 2015

(C) Korean Society of Nuclear Medicine 2015

\section{Editor-in-Chief}

In the last issue, I announced a new editorial board that can deal with every aspect of nuclear medicine, and especially the prospects of our discipline in the future. We do basic research not for the sake of science itself but for the purpose of meeting clinical needs. During the last several decades nuclear medicine has prospered, with the help and support of related sciences and engineering. These sciences were collectively called 'biology,' and because of that, the global or continental efforts were referred to as 'Nuclear Medicine and Biology,' as was implemented in the names of Federations such as the WFNMB (World Federation of Nuclear Medicine and Biology) or AOFNMB (Asia and Oceania Federation of Nuclear Medicine and Biology). Radiochemistry, radiopharmacy, or radiopharmaceutical sciences have supplied chemistry platforms for nuclear medicine since its birth and have majored in developing small molecules, peptides, and biomacromolecules. The most brilliant star of the twentieth century was F-18 fluorodeoxyglucose, and peptides were successfully used for radionuclide targeted therapy. The advent of monoclonal antibodies was the cause of hype over the 1990s, and the terms 'radioimmunoscintgraphy' and 'radioimmunotherapy' were devised. However, the enthusiasm did not persist long, because after the serious successful clinical trials and introduction of I-131 tositumomab $\left(\right.$ Bexxar $\left.^{\circledR}\right)$, pharmaceutical companies lost interest within a short while and left the drug as an 'orphan'. On the other hand, Lu-177 or Y-90 labelled DOTATOC and DOTATATE therapy

Dong Soo Lee

dsl@plaza.snu.ac.kr

1 Department of Nuclear Medicine, Seoul National University, 101 Daehang-ro, Jongro-gu, Seoul 110-744, Korea

was firmly introduced to the clinics by European colleagues. This therapy was characterized as theranostics interestingly because the therapy response was evaluated with accompanying nuclear imaging using SPECT (Lu-177) or PET (Y-90). Theranostics promises the evaluation of the therapeutic effect and also the prediction of therapeutic efficacy.

In addition to the use of small molecules to biomacromolecules, particulate materials were recently proposed to be used as platforms for radiolabelling and theranostics. This progress is mainly due to the success of our chemists/chemical engineers in producing nanomaterials and controlling the uniformity and uniqueness of these nanomaterials. In the past, the scientific community did not understand how to synthesize these nano-sized particulate molecules and modify their surface. Once they understood this, they immediately offered the use of nanomaterials for imaging and therapy. In retrospect, imaging was tried with quantum dots or carbon nanotubes and therapy was tried with gold particles with photothermal therapy. However, quantum dot use was plagued with toxicity woes due to cadmium, and carbon nanotubes were proven to be very toxic to the lungs, just like asbestos or silica. The surface reactivity of noble metals such as gold and silver was not 'noble'. While they are in their nano-sized dimension, they are no longer 'nobly inert metals' but are very much reactive. Now nuclear medicine is about to take advantage of the characteristics of nanomaterials (which are also called nanoparticles or nanostructures) for imaging and therapy. This has recently been called 'radionanomedicine,' representing the combined use of nuclear medicine and nanomedicine [1]. Radionanomedicine is intended to be radio-theranostics with nanomaterial platforms.

The adoption of nanomedicine to nuclear medicine will be accompanied by successful clinical application or otherwise be dormant for a long time in its basic research epoch. We now 
do not know if the rise of nanomedicine is temporary hype or is the hope for the expanded theranostics of nuclear medicine. Recently, I proposed that the use of nanomedicine should adopt the core of nuclear medicine technology, which is tracer technology. Tracer technology consists of use of trace amounts and tracing the in vivo biodistribution, which means imaging.

In the next issues, I will try to deal with the scope of nuclear medicine theranostics, which is based upon the technology applied for radionanomedicine. Surface modification chemistry and the combination of targeting ligands, peptides and antibodies on the surface for multiplexing are the main breakthroughs of this field. Also, the supply of nanoplatforms will enhance the implementation of nuclear medicine theranostics or radionanomedicine [2]. Again, chemistry will be devoted to the proliferation of nuclear medicine. I envision that the chemical or nanotechnological aid might be more influential for the future of nuclear medicine than biological or molecular biological perspectives have influenced the rational application of nuclear medicine to humans. We, the nuclear medicine experts, are going to see what happens next or are more than willing to realize its early implementation.

Conflict of Interest Dong Soo Lee declare no conflict of interest.

\section{References}

1. Lee DS, Im HJ, Lee YS. Radionanomedicine: widened perspectives of molecular theragnosis. Nanomedicine. 2015;11:795-810.

2. Lee YS, Kim YI, Lee DS. Future perspectives of the chemistry for radionanomedicine. Nucl Med Mol Imaging. 2015;49 in press. 